\title{
Recurrent Cerebellar Hemangioblastoma with Thoracic Intramedullary Spinal Tumor in a Young Patient with Von Hippel Lindau Disease: A Case Report
}

\section{K Raja Shekar* and BT Badadal}

Consultant Neurosurgeon, Department of General Surgery, Shri B.M Patil Medical

College and Hospital and Research Center, Vijayapura, Karnataka, India

*Corresponding Author: K Raja Shekar, Consultant Neurosurgeon, Department of General Surgery, Shri B.M Patil Medical College and Hospital and Research Center, Vijayapura, Karnataka, India.
Received: May 29, 2021

Published: June 23, 2021

(C) All rights are reserved by K Raja Shekar and BT Badadal.

\section{Abstract}

Introduction: Hemangioblastomas are most common intraaxial tumors in patients with VHL syndrome.

They are commonly seen in cerebellum and spinal cord. These are associated with retinal angiomas, cystic lesions in pancreas, kidney and epididymis.

Case Presentation: A 22 year old man who underwent surgery for cerebellar hemangioblastoma 4 years ago has now presented with complaints of headache, visual blurring, difficulty in walking since 2 months. He also had decreased speech output.

His family history was significant as his mother died of cerebellar hemangioblastoma.

MRI showed right cerebellar cystic lesion with enhancing mural nodule with moderate hydrocephalus (Figure 1) and dorsal intramedullary spinal lesion extending from T1 to T5 (Figure 2).

Fundoscopy examination showed retinal angioma of left eye. ultrasound abdomen and pelvis was normal.

Treatment: Revision suboccipital craniectomy was performed and gross total excision of cyst with mural nodule was done.

Histopathology confirmed the diagnosis of hemangioblastoma.

Patient improved clinically and was discharged 5 days after surgery.

Conclusion: Hemangioblastomas have high tendency for recurrence if GTE is not done. Screening of whole spine with fundoscopy is mandatory for patients with VHL.

Keywords: VHL: Von Hippel Lindau; GTE: Gross Total Excision; CNS: Central Nervous System; MRI: Magnetic Resonance Imaging; USG: Ultrasonogram

\section{Introduction}

Von Hippel-Lindau syndrome (VHL) is a hereditary condition associated with tumors arising in multiple

organs. VHL-related tumors include hemangioblastomas of the brain, spinal cord, and retina.
Other features of VHL include

Pheochromocytomas of adrenal gland, kidney cysts; pancreatic cysts, epididymal cystadenomas, broad ligament cystadenomas, and endolymphatic sac tumors.

\section{Case Presentation}

A 22 year old man who underwent surgery for cerebellar hemangioblastoma 4 years ago has now presented with complaints of 
headache, visual blurring, difficulty in walking since 2 months. He also had decreased speech output.

His family history was significant as his mother died of cerebellar hemangioblastoma.

MRI showed right cerebellar cystic lesion with enhancing mural nodule with moderate hydrocephalus (Figure 1) and dorsal intramedullary spinal lesion extending from T1 to T5 (Figure 2).

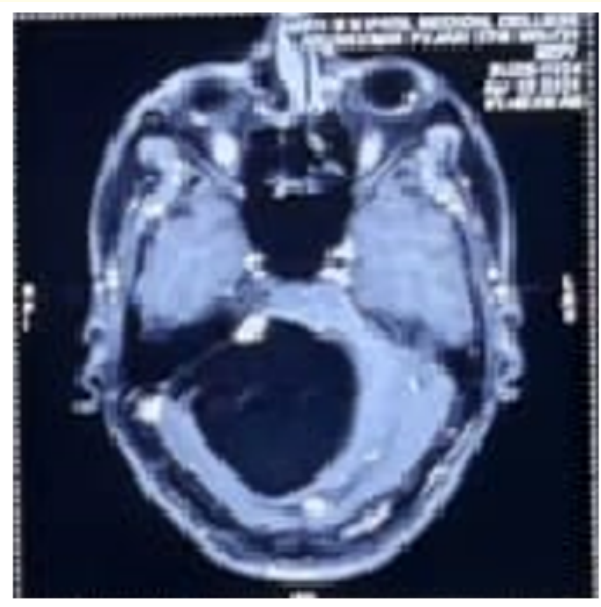

Figure 1: Gadalonium contrast MRI showing right cerebellar cystic tumor with mural nodule.

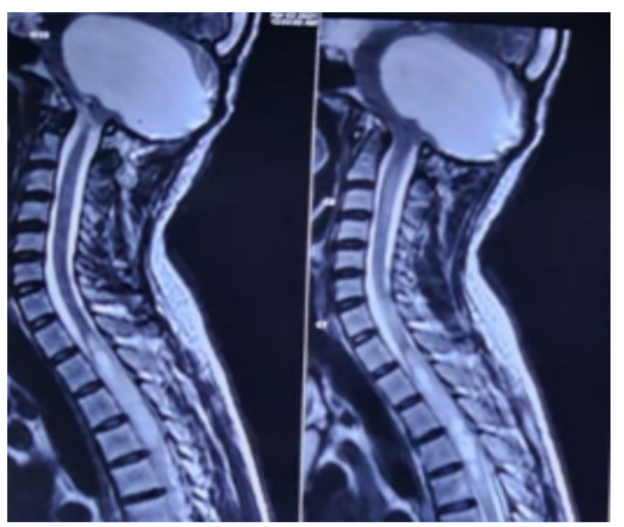

Figure 2: T2 sagittal MRI scan showing spinal cord intramedullary lesion extending from $\mathrm{T} 1$ to T5 level.

\section{Discussion}

Haemangioblastomas are benign central nervous system tumours comprising vascular endothelial and stromal components.
They occur sporadically or in association with VHL. These tumours do not always exhibit a linear growth pattern, instead often demonstrating periods of accelerated growth interrupted by episodes of relative stasis. Microsurgical resection is the treatment of choice for most tumours and is considered safe in experienced hands [1].

Spinal haemangioblastomas are intradural intramedullary tumours and most are found at the level of the cervical and dorsal vertebrae [2]. In our case the spinal lesion extended from $\mathrm{T} 1$ to T5. Spinal cord hemangioblastomas comprise $2-3 \%$ of primary intramedullary spinal cord tumors and are the third most common among them [3]. It has been documented that an age of less than 30 years on initial diagnosis, the presence of von Hippel-Lindau disease, and a multicentric involvement of the central nervous system are all independent predictors of tumour recurrence [4].

\section{Surveillance}

CNS imaging for intercurrent haemangioblastomas should be done yearly. Poulsen showed that annual CNS examinations reduced the risk of an intercurrent manifestation to $2.7 \%$ compared to $7.2 \%$ with biennial examinations [5].

In our case gross total excision of tumor was done (Figure 3) which was confirmed by post operative CT scan (Figure 4).

\section{Conclusion}

VHL disease should be considered as one of the most important differential diagnosis for cerebellar hemangioblastoms.

The disease spectrum of VHL requires through screening and surveillance along with prompt surgical excision.

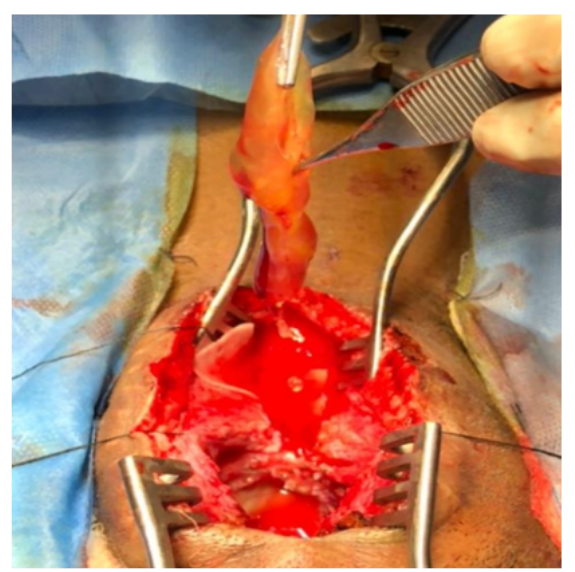

Figure 3: Intraoperative image demonstrating cystic tumor. 


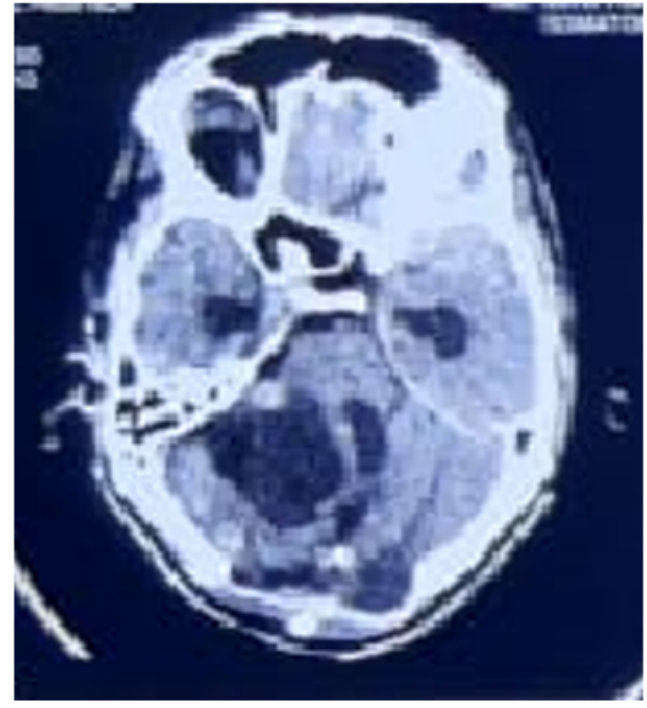

Figure 4: Postoperative CT scan showing gross total excision of tumor.

\section{Conflict of Interest}

NO.

\section{Informed Consent Taken from Patient}

Yes.

\section{Bibliography}

1. Lonser Russell R., et al. "von Hippel-Lindau disease". The Lancet 361.9374 (2003): 2059-2067.

2. Beitner M M., et al. "Neurosurgical considerations in von Hippel-Lindau disease". Journal of Clinical Neuroscience 18.2 (2011): 171-180.

3. Park Deric M., et al. "von Hippel-Lindau disease-associated hemangioblastomas are derived from embryologic multipotent cells". PLoS Medicine 4.2 (2007): e60.

4. de la Monte Suzanne M and Stephanie A Horowitz. "Hemangioblastomas: clinical and histopathological factors correlated with recurrence". Neurosurgery 25.5 (1989): 695-698.
5. Poulsen M L M., et al. "Surveillance in von Hippel-Lindau disease (VHL)". Clinical Genetics 77.1 (2010): 49-59.

\section{Volume 4 Issue 7 July 2021}

(c) All rights are reserved by K Raja Shekar and BT Badadal. 